\title{
DIFFERENTIAL OPERATORS ON THE ALGEBRA OF DENSITIES AND FACTORIZATION OF THE GENERALIZED STURM-LIOUVILLE OPERATOR
}

\author{
EKATERINA SHEMYAKOVA AND THEODORE VORONOV
}

\begin{abstract}
We consider factorization problem for differential operators on the commutative algebra of densities (defined either algebraically or in terms of an auxiliary extended manifold) introduced in 2004 by Khudaverdian and Voronov in connection with BatalinVilkovisky geometry. We consider the case of the line, where unlike the familiar setting (where operators act on functions) there are obstructions for factorization. We analyze these obstructions. In particular, we study the "generalized Sturm-Liouville" operators acting on the algebra of densities on the line. This in a certain sense is in between the $1 \mathrm{D}$ and $2 \mathrm{D}$ cases. We establish a criterion of factorizabily for the generalized Sturm-Liouville operator in terms of solution of the classical Sturm-Liouville equation. We also establish the possibility of an incomplete factorization.
\end{abstract}

\section{INTRODUCTION}

In this letter we consider differential operators on the algebra of densities. The algebra of densities is a commutative algebra of functions of special form defined on a formally extended (super)manifold, where a formal differentiation relative to the extra variable provides a new convenient way to consider differential operators acting on densities of particular weights together. But also note that the original motivation for the development of the algebra of densities was the geometry of Batalin-Vilkovisky operators, see the original paper [16].

It has turned out that one may classify its derivations (obtaining an analog of Nijenhuis's theorem about derivations of differential forms), describe Poisson brackets, etc. It is also related with classical constructions of differential geometry such as projective connections and the notion of Thomas's bundle. See [17, 18, [8], 2], and [15].

The interest in differential operators acting on densities comes from physics and also from integrable systems, and the work towards understanding general structure of such operators has started in 90s. Some classification results were obtained for the spaces of differential operators of certain types acting on densities of different weights and considered as modules over Lie algebras of vector fields, and also for the mappings that relate these spaces, e.g. [5, 7].

One of the works we pay more attention to in this note is Duval and Ovsienko [5], where as part of such a classification of operators of order two and of weight zero, they discovered "singularities" at weights $\lambda=0,1 / 2,1$. An explanation of that was obtained in [3] in terms of canonical pencils associated with self-adjoint operators on the algebra of densities introduced in [16] and motivated by BV geometry. Duval and Ovsienko [5] also contains a formula for the (unique) equivariant mapping connecting those spaces of differential

Date: 15 (28) June 2018. 
operators (as modules over vector fields), which led us into a new natural derivation of the algebra of densities and a connection with this series of works, see in Sec. 2.4.

Factorization is a fundamental problem for ordinary and partial differential operators. For operators in 1D, it is known that a factorization is always possible (in a large enough differential field extension) and is closely related with solution of the corresponding differential equations. Starting from 2D, factorization is rarely possible. Obstructions to factorizations describe algebraic properties of the operators and are related with other important questions. For example, the authors' particular further interest is the existence and types of Darboux transformations (see e.g. [24, 23], [11, [22]). The case of differential operators on the algebra of densities on the line is in a certain sense "in between" the 1D and $2 \mathrm{D}$ cases, so it is interesting to see how an obstruction to factorization may arise. We show that unlike the $1 \mathrm{D}$ case, the operators on the algebra of densities $\mathfrak{D} \mathfrak{e n s}(\mathbb{R})$ are not always factorizable. Our main result is for generalized Sturm-Liouville operator, which is a self-adjoint second order differential operator on the algebra $\mathfrak{D} \mathfrak{e n} \mathfrak{s}(\mathbb{R})$ on the line that specializes to the classical Sturm-Liouville when restricted on densities of weight $-1 / 2$. We establish a criterion for factorization of the generalized Sturm-Liouville operator. It is formulated in terms of a density invariant (that we found) of the generalized SturmLiouville operator, and the criterion is that this density invariant should be a solution of the corresponding classical Sturm-Liouville equation. We also establish the possibility of an incomplete factorization, which is unique.

This letter reports the first step in the project of unifying the study of algebraic properties of differential operators including factorization and Darboux transformations with their geometric analysis. Although the idea of the algebra of densities and differential operators on it first appeared in the context of supergeometry (from the study of odd Laplace operators), here we do not consider the super case, which we hope to address in the future (and to compare it with [19]).

\section{Differential operators on the Algebra of Densities}

The algebra of densities was discovered by Khudaverdian and Voronov in [16] while studying geometric structures related with the Batalin-Vilkovisky formalism. Here we recall basic facts about this algebra, including differential operators on it and their interpretation in terms of the extended manifold, see [16, 18]. The generalized Sturm-Liouville operator on the algebra of densities will be introduced in Sec. 4.2.

2.1. Densities and the algebra of densities. Scalar and tensor densities are well known in differential geometry and applications in physics. We shall deal with scalar densities. A (scalar) density of weight $\lambda \in \mathbb{R}$ on a (super)manifold $M$ with local coordinates $x^{a}$ is a formal expression of the form $\rho=\rho(x)|D x|^{\lambda}$. The formal symbol $|D x|$ under a change of coordinates transforms by

$$
|D x|=\left|D x^{\prime}\right|\left|\frac{D x}{D x^{\prime}}\right|
$$

and its formal power $|D x|^{\lambda}$ transforms by

$$
|D x|^{\lambda}=\left|D x^{\prime}\right|^{\lambda}\left|\frac{D x}{D x^{\prime}}\right|^{\lambda} .
$$


Here we use the classical notation $D x / D x^{\prime}$ for the Jacobian, so

$$
\frac{D x}{D x^{\prime}}=\operatorname{det} \frac{\partial x}{\partial x^{\prime}}
$$

in the ordinary case and

$$
\frac{D x}{D x^{\prime}}=\operatorname{Ber} \frac{\partial x}{\partial x^{\prime}}
$$

in the supercase. (The main results of this paper are not concerned with supermanifolds, but we mention the supercase here for completeness; also, the algebra of densities, see below, arose first in the super context for the purposes of Batalin-Vilkovisky formalism.) The line bundle whose sections are $\lambda$-densities is trivial topologically but is not trivialized canonically unless a special structure such as a volume element is fixed. A density of weight 0 is a function. A density of weight 1 is a (non-oriented) volume form, so the integral $\int_{M} \rho(x)|D x|$ over the manifold is well-defined, i.e., independent of a choice of coordinates; no orientation is required. (One needs compact supports of course.) For integral weights $\lambda=0, \pm 1, \pm 2, \ldots$, densities are sections of tensor powers of the line bundle of volume forms. Densities of other weights arise naturally in applications. Wave functions $\psi$ in quantum mechanics are densities of weight $\frac{1}{2}$ because the squares of their absolute values $|\psi|^{2}$ have the meaning of probability densities and so are densities of weight 1 . In an example that we shall consider, we shall naturally encounter densities of weights such as $-\frac{1}{2}$ and $\frac{3}{2}$. Notation: $\mathfrak{D e n s}_{\lambda}(M)$ for the space of densities of weight $\lambda$ on $M$.

Densities can be multiplied, so that the weights add up: if $\psi=\psi(x)|D x|^{\lambda}$ and $\chi=$ $\chi(x)|D x|^{\mu}$, then $\psi \chi=\psi(x) \chi(x)|D x|^{\lambda+\mu}$. In [16], it was observed that considering the direct sum

$$
\mathfrak{D e n s}(M):=\bigoplus_{\lambda \in \mathbb{R}} \mathfrak{D} \mathfrak{e n s}_{\lambda}(M)
$$

as an algebra under such a multiplication extended by linearity to formal sums of densities of different weights gives an advantage similar to that obtained by considering the algebra of differential forms. The algebra of densities $\mathfrak{D} \mathfrak{e n s}(M)$ so defined can be analyzed in an abstract form as a commutative algebra with unit (e.g. considering its derivations and differential operators in the algebraic sense), as well as a particular algebra of functions (see more in the next subsection) where geometric intuition can be applied. The algebra $\mathfrak{D e n s}(M)$ includes the algebra of functions, $C^{\infty}(M)=\mathfrak{D e n s}_{0}(M)$, as a subalgebra, hence it in particular contains constants and the unit 1.

The integral $I=\int_{M}$ can be extended by zero to densities of all weights $\neq 1$, giving a linear functional $I: \mathfrak{D} \mathfrak{e n s}(M) \rightarrow \mathbb{R}$, which allows to extend the non-degenerate pairing of the spaces $\mathfrak{D e n s}_{\lambda}(M)$ and $\mathfrak{D e n s}_{1-\lambda}(M)$ (one can consider $\mathfrak{D e n s}_{1-\lambda}(M)$ as the "smooth subspace" of the dual $\left.\left(\mathfrak{D e n s}_{\lambda}(M)\right)^{*}\right)$ to an invariant scalar product on $\mathfrak{D} \mathfrak{e n s}(M)$,

$$
\langle\psi, \chi\rangle:=I(\psi \chi),
$$

where, strictly speaking, we consider the subalgebra of densities with compact support. More specifically, for $\psi \in \mathfrak{D e n s}_{\lambda}(M)$ and $\chi \in \mathfrak{D e n s}_{\mu}(M)$, define

$$
\langle\psi, \chi\rangle:= \begin{cases}\int_{M} \psi \chi, & \lambda+\mu=1 \\ 0 & \text { otherwise }\end{cases}
$$

and then extend by bilinearity. 
2.2. Densities as functions on the extended manifold. The algebra $\mathfrak{D e n s}(M)$ can be interpreted [16] as a subalgebra of the algebra of functions on an extended manifold $\hat{M}$, the total space of the frame bundle for the line bundle $|\operatorname{Ber} T M|$. The symbol $|D x|$ can be taken as a basis element in the line bundle $\left|\operatorname{Ber} T^{*} M\right|$, which is dual to $|\operatorname{Ber} T M|$. It can be replaced by an invertible auxiliary variable $t$. The variables $x^{a}, t$ are local coordinates on $\hat{M}$ with the transformation law $x^{a}=x^{a}\left(x^{\prime}\right), t=|J| t^{\prime}$, where $J=\operatorname{Ber} \frac{\partial x}{\partial x^{\prime}}$. Therefore an element of $\mathfrak{D e n s}(M)$ corresponds to a function of $x^{a}, t$,

$$
\psi=\sum \psi_{\lambda}(x)|D x|^{\lambda} \longleftrightarrow \psi(x, t)=\sum \psi_{\lambda}(x) t^{\lambda},
$$

a "pseudo-polynomial" in $t$. By construction, the subalgebra $\mathfrak{D e n s}(M) \subset C^{\infty}(\hat{M})$ is graded (by real numbers), unlike the whole algebra $C^{\infty}(\hat{M})$.

Remark 1. The fiber bundle $\hat{M} \rightarrow M$ was used in classical differential geometry by T. Y. Thomas [25] for description of projective connections on a manifold $M$. The auxiliary manifold $\hat{M}$ is therefore sometimes referred to as the Thomas manifold or Thomas bundle. See also [8]. Unlike in that classical application, here the Thomas manifold $\hat{M}$ is taken together with the graded algebra $\mathfrak{D e n s}(M)$ as its algebra of functions and hence is regarded as a "graded manifold".

Linear operators on the algebra $\mathfrak{D e n s}(M)$ and other objects associated with it naturally assume grading. They can be regarded as geometric objects on the graded manifold $\hat{M}$. Such are, for example, the graded derivations of $\mathfrak{D} \mathfrak{e n s}(M)$ (equivalently, graded vector fields on $\hat{M}$ ), which are classified in [16] (see also [18]).

2.3. Differential operators on the algebra of densities. Differential operators on $\mathfrak{D e n s}(M)$ can be introduced algebraically or in terms of local coordinates $x^{a}, t$ on $\hat{M}$. Consider the operator $\hat{w}$ defined by $\hat{w}(\rho)=\lambda \rho$ for $\rho \in \mathfrak{D e n s}_{\lambda}(M)$. It is a derivation of $\mathfrak{D e n s}(M)$ and so a differential operator of order 1. It is called the weight operator. (Note a similarity with the number operator in quantum field theory.) In coordinates,

$$
\hat{w}=t \frac{\partial}{\partial t} \text {. }
$$

A linear operator $L$ on $\mathfrak{D e n s}(M)$ has weight $\mu$, i.e., for all $\lambda, L$ maps $\mathfrak{D e n s}_{\lambda}(M)$ to $\mathfrak{D e n s}_{\lambda+\mu}(M)$, if

$$
[\hat{w}, L]=\mu L .
$$

In particular, $\hat{w}$ itself has weight 0 and because $t$ is invertible, the partial derivative in $t$ can be expressed via $\hat{w}$ as

$$
\frac{\partial}{\partial t}=t^{-1} \hat{w}
$$

Therefore it is convenient to write homogeneous differential operators on the algebra $\mathfrak{D e n s}(M)$ as

$$
L=t^{\mu} \sum_{p+|q| \leq n} a_{p q}(x) \hat{w}^{p} \partial_{x}^{q},
$$

where $\partial_{x}=\frac{\partial}{\partial x^{a}}$ and $q$ is a multi-index. Formula (5) gives the (local) general form of a differential operator of order $\leq n$ and weight $\mu$ on the algebra $\mathfrak{D e n} \mathfrak{e}(M)$. A special case 
of (41) is the commutation relation

$$
\left[\hat{w}, t^{\mu}\right]=\mu t^{\mu}
$$

for the operator of multiplication by $t^{\mu}$. Note also the formulas for transformation under a change of coordinates $x^{a}=x^{a}\left(x^{\prime}\right), t=t^{\prime}|J|$ : the operator $\hat{w}$ is invariant, and the partial derivatives transform as

Here $J=\operatorname{Ber} \frac{\partial x}{\partial x^{\prime}}$.

$$
\frac{\partial}{\partial x^{a}}=\frac{\partial x^{a^{\prime}}}{\partial x^{a}} \frac{\partial}{\partial x^{a^{\prime}}}-\frac{\partial \ln |J|}{\partial x^{a}} \hat{w} .
$$

When a homogeneous operator on $\mathfrak{D} \mathfrak{e n s}(M)$ is restricted on direct summands $\mathfrak{D} \mathfrak{e n s} \mathfrak{s}_{w}(M)$, for all values of $w \in \mathbb{R}$, it gives a family of differential operators $\mathfrak{D e n s}_{w}(M) \rightarrow \mathfrak{D e n s}_{w+\mu}(M)$, where $\mu$ is the weight of $L$. Following [16], we call it the operator pencil associated with $L$,

$$
L_{w}=t^{\mu} \sum_{p+|q| \leq n} a_{p q}(x) w^{p} \partial_{x}^{q} .
$$

The order of a pencil is counted jointly by the powers of $w$ and the order of the derivatives.

Example 1. An operator $L$ of order 1 on $\mathfrak{D e n s}(M)$ corresponds to a linear pencil $L_{w}$ :

$$
L=t^{\mu}\left(A^{a}(x) \partial_{a}+B(x) \hat{w}+C(x)\right) \longleftrightarrow L_{w}=|D x|^{\mu}\left(A^{a}(x) \partial_{a}+B(x) w+C(x)\right) .
$$

Here $\partial_{a}=\frac{\partial}{\partial x^{a}}$.

When convenient, we may drop an indication to the manifold and write $\mathfrak{D e n s}$ or $\mathfrak{D e n s} \mathfrak{s}_{\lambda}$.

2.4. Derivation of the algebra of densities from a different perspective. The algebra of densities [16] was found in 2004 as the "correct" framework for the BatalinVilkovisky $\Delta$-operator that uncovers the geometry of this operator. The BV $\Delta$-operator on an odd symplectic manifold had generally been thought to be acting on functions and its definition required a choice of a volume element [12], [21], until it was discovered in 1999 that the $\Delta$-operator is canonically defined on semidensities [13, 14] without imposing any additional structure. Further investigations led from semidensities to the algebra of densities in [16, where the long-standing problem of describing all operators generating a given "bracket" was fully solved. Hörmander's sub-principal symbol naturally fit into this framework as a "connection-type" geometric object (for densities of a fixed weight) and a part of the principal symbol on the extended manifold $\hat{M}$.

In this letter, we are interested in other problems, but we have adopted the algebra of densities as a convenient tool for working with differential operators acting on densities. Below we connect this approach with a large series of works by several authors who studied interrelation between modules of differential operators acting on densities of different weights. A notable paper in this series is Duval and Ovsienko [5], which considers the space $\mathcal{D}_{\lambda}^{2}$ of second-order linear differential operators of weight zero acting on densities of some particular weight $\lambda$,

$$
L=a_{2}^{i j} \partial_{i} \partial_{j}+a_{1}^{i} \partial_{i}+a_{0}, \quad L: \mathfrak{D e n s}_{\lambda} \rightarrow \mathfrak{D e n s}_{\lambda}
$$

as a module over the Lie algebra of vector fields. It turned out that for most values of $\lambda$, all the modules $\mathcal{D}_{\lambda}^{2}$ are isomorphic to each other. The only "singular" values of $\lambda$ are 
$\lambda=0,1 / 2,1$ for $\operatorname{dim} M \geq 2$ and $\lambda=0,1$ for $\operatorname{dim} M=1$. It was proved that there is a unique (up to a constant) equivariant linear mapping

$$
\mathcal{L}_{\lambda \mu}^{2}: \mathcal{D}_{\mu}^{2} \rightarrow \mathcal{D}_{\lambda}^{2}
$$

and coordinate formulas for it were found. In particular, for $\operatorname{dim} \geq 2$, the transformation $\mathcal{L}_{\lambda \mu}^{2}$ for non-singular weights $\lambda$ and $\mu$ is given by

$$
\begin{aligned}
& \widetilde{a}_{2}^{i j}=a_{2}^{i j}, \\
& \widetilde{a}_{1}^{l}=\frac{2 \lambda+1}{2 \mu+1} a_{1}^{l}+2 \frac{\mu-\lambda}{2 \mu+1} \partial_{i} a_{2}^{i l}, \\
& \widetilde{a}_{0}=\frac{\lambda(\lambda+1)}{\mu(\mu+1)} a_{0}+\frac{\lambda(\mu-\lambda)}{(2 \mu+1)(\mu+1)}\left(\partial_{i} a_{1}^{i}-\partial_{i} \partial_{j} a_{2}^{i j}\right)
\end{aligned}
$$

(formulas do not depend on a choice of a local coordinate system). These Duval-Ovsienko results can be used for the following argument. Since all the modules $\mathcal{D}_{\lambda}^{2}$ corresponding to non-singular values of $\lambda$ are isomorphic, one is naturally led to the idea that their elements should be studied as single objects depending on $\lambda$ as a parameter. So, if we fix some nonsingular value of $\mu$ and some operator $L \in \mathcal{D}_{\mu}^{2}$, then by applying to it the Duval-Ovsienko transformation $\mathcal{L}_{\lambda \mu}$, we arrive at an operator pencil $L_{\lambda}$ (i.e., a one-parameter family of operators $L_{\lambda} \in \mathcal{D}_{\lambda}^{2}$ ). The general structure of the formula for $L_{\lambda}$ is that the coefficients at $\partial_{i} \partial_{j}$ do not contain $\lambda$, the coefficients at $\partial_{i}$ are linear in $\lambda$ and the free term coefficients are quadratic in $\lambda$. Therefore, our "true objects" obtained this way are characterized by the total order in the derivatives $\partial_{i}$ and the parameter $\lambda$ together (which should be $\leq 2$ ).

Now, if we return to the algebra of densities, we see that to every differential operator on the algebra $\mathfrak{D} \mathfrak{e n s}$ corresponds an operator pencil (see (8) ) which has the desired structure of the coefficients automatically. Thus, if we write a second order operator on $\mathfrak{D e n s}$ as

$$
\widehat{L}=A_{2}^{i j} \partial_{i} \partial_{j}+A_{1}^{i} \partial_{i}+A_{0}: \mathfrak{D e n s} \rightarrow \mathfrak{D e n s}
$$

where $A_{2}^{i j}$ does not contain $\widehat{w}, A_{1}^{i}$ is of order $\leq 1$ in $\widehat{w}, A_{0}$ is of order $\leq 2$ in $\widehat{w}$, and restrict the operator $\widehat{L}$ onto $\mathfrak{D e n s}_{\lambda}$, we shall obtain differential operators $\mathfrak{D} \mathfrak{e n \mathfrak { s } _ { \lambda }} \rightarrow \mathfrak{D} \mathfrak{e n \mathfrak { s } _ { \lambda }}$, for each $\lambda$, whose coefficients depend on local coordinates $x^{i}$ and are polynomial in $\lambda$ with the same property as above.

Furthermore: the above procedure based on Duval-Ovsienko formulas gives operator pencils that not only have the dependence on the parameter corresponding to second-order differential operators on the algebra $\mathfrak{D e n s}$, but a closer look at the formulas shows that these second-order operators on $\mathfrak{D e n s}$ also happen to be self-adjoint (i.e., exactly those studied in [16]). This was observed in [3]. The interesting question of what is happening at a singular value of parameter is considered in [15].

\section{Factorization of Differential operators on the Algebra $\mathfrak{D e n s}(\mathbb{R})$}

3.1. Factorization of differential operators in the classical situation. Here we briefly overview the factorization problem for differential operators in the classical setup. In it, it is assumed that the operators act on functions, but behaviour under changes of independent variables is not emphasized, and the geometric nature of objects on which the operators act is not really important in this context. We shall recall that the onedimensional case and the higher dimensional case are very different. 
To illustrate the situation in the one-dimensional case, consider the simplest possible example of the operator $L=\partial^{2}$. It has a commutative factorization $L=\partial \circ \partial$ and a one-parameter family of non-commutative factorizations

$$
L=\partial^{2}=\left(\partial+\frac{1}{x+c}\right) \circ\left(\partial-\frac{1}{x+c}\right) .
$$

One can show that these are the only possible factorizations. In general, for an operator $L=a_{n} \partial^{n}+a_{n-1} \partial^{n-1}+\cdots+a_{1} \partial+a_{0}$, where $a_{i}$ do not need to be constants, there is Frobenius's theorem [6] stating that factorizations into first-order factors are in one-to-one correspondence with maximal flags in the kernel of $L$. For example, continuing with the operator $L=\partial^{2}$, one gets for a particular flag

$$
\langle x\rangle \subseteq\langle x, 1\rangle=\operatorname{ker} L \quad \longleftrightarrow \quad L=\left(\partial+\frac{1}{x}\right) \circ\left(\partial-\frac{1}{x}\right)
$$

which can be computed as $L=L_{2} \circ L_{1}$, where $L_{1}=x \circ \partial \circ(x)^{-1}$ and then $\varphi_{2}=L_{1}(1)=-1 / x$, and $L_{2}=\varphi_{2} \circ \partial \circ \varphi_{2}^{-1}$.

Already in two dimensions, the situation is radically different, as shown by the famous example of E. Landau: for $P=\partial_{x}+x \partial_{y}, Q=\partial_{x}+1, R=\partial_{x}^{2}+x \partial_{x} \partial_{y}+\partial_{x}+(2+x) \partial_{y}$ (irreducible in any reasonable extension), we have $Q Q P=R Q$, which means that an operator of third order has two completely different factorizations into irreducible factors. Noteworthy, even the numbers of the irreducible factors are different. In general, in the multidimensional case there is no general theory, but only some results for particular situations that are available.

Factorization of differential operators is a fundamental problem in mathematics, and there are, very broadly speaking, three main aspects here: analytic (where the objects on which the operators act are analyzed as functions satisfying this or that analytic properties), algebraic and computational (where the objects are treated as elements of some differential field and so all the questions about smoothness, etc., are skipped), and geometric (where the geometric nature of objects is important, e.g. scalar functions, densities, differential forms, etc.).

Our interest here is geometric and we shall be concerned with the factorization problem in the framework of the algebra of densities. In [9, 19], among other results we considered factorization of differential operators on the superline (i.e., a manifold of dimension 1|1). It turned out that for non-degenerate operators of arbitrary order an analog of the Frobenius theorem holds. Further, in [9, 19], it was shown that non-degenerate differential operators in dimension 1|1 are similar to operators in the one-dimensional case.

\subsection{Factorization of differential operators on the algebra of densities. Obstruc-}

tion. If we restrict ourselves to the algebra $\mathfrak{D e n s}(\mathbb{R})$ and operators of order two on it (which we think is not such a restriction as the ideas for operators of orders larger than two probably will be similar to the ones of order two), we shall discover that the situation with factorization of differential operators is very different here. 
Now, the most general form for factorization here will be as follows:

$$
\begin{aligned}
L & =t^{2}\left(\partial^{2}+\left(p_{1} \hat{w}+p_{0}\right) \partial+q_{2} \hat{w}^{2}+q_{1} \hat{w}+q_{0}\right) \\
& =t\left(\partial-\alpha_{1} \hat{w}-\alpha_{0}\right) \cdot t\left(\partial-\beta_{1} \hat{w}-\beta_{0}\right) \\
& =t^{2}\left(\partial-\alpha_{1}(\hat{w}+1)-\alpha_{0}\right) \cdot\left(\partial-\beta_{1} \hat{w}-\beta_{0}\right),
\end{aligned}
$$

where $p_{i}, q_{i}, \alpha_{i}, \beta_{i}$ are functions of $x$ and where the commutation rule $\hat{w} t^{\lambda}=t^{\lambda}(\hat{w}+\lambda)$ was used in the last line. The negative signs are for convenience of further computations. Note that we consider monic operators of matching weights to ensure invariance under changes of coordinates.

Up to the factor of $t^{2}$, this may be viewed as a problem of factoring a univariate differential operator acting on functions of $x$, with coefficients depending on a parameter $w$, of a special form dictated by the homogeneity condition. So, morally it is "in between" 1D and $2 \mathrm{D}$ cases.

To explore the similarity with the 1D case, we switch to a condensed notation $\hat{\alpha}=$ $-\alpha_{1}(\hat{w}+1)-\alpha_{0}, \hat{\beta}=-\beta_{1} \hat{w}-\beta_{0}, \hat{p}=p_{1} \hat{w}+p_{0}, \hat{q}=q_{1} \hat{w}+q_{0}$, which hides dependence on $\hat{w}$ and makes the problem look similar to the problem in the classical setting:

$$
L=t^{2}\left(\partial^{2}+\hat{p} \partial+\hat{q}\right)=t^{2}(\partial-\widehat{\alpha}) \cdot(\partial-\widehat{\beta})
$$

This implies $\widehat{\alpha}=-\hat{p}-\widehat{\beta}$ and the familiar Riccati equation for $\widehat{\beta}$ :

$$
-\widehat{\beta}^{\prime}=\widehat{\beta}^{2}+\hat{p} \widehat{\beta}+\hat{q} \text {. }
$$

Warning: here we are applying prime for the derivative in $x$ (and not for transformation of variables!) for a more expedient notation. In the classical case, substitution $\widehat{\beta}=\partial(\ln \varphi)$ is used to transform the Riccati equation into

$$
\varphi^{\prime \prime}+\hat{p} \varphi^{\prime}+\hat{q} \varphi=0 .
$$

That leads to the known fact that in the classical situation, every $\varphi$ in the kernel of $L$ implies a factorization of $L=\partial^{2}+\hat{p} \partial+\hat{q}$. So in a suitable field of coefficients all 1D differential operators are factorizable.

In the densities case, the operator is factorizable only if there is a solution $\varphi$ of (15) of a certain special form, namely, such that $\widehat{\beta}$ computed as $\widehat{\beta}=\partial(\ln \varphi)$ is linear in $\hat{w}$.

Example 2. The following operator on the algebra of densities (which is a generalized Sturm-Liouville operator, see next section) is not factorizable:

$$
L=t^{2} \cdot\left(\partial^{2}-\hat{w}^{2}-\hat{w}\right) .
$$

See more in Sec. 4.3 .

Let us now return to the non-condensed notation, and look more closely at this Riccati. We had

$$
\begin{aligned}
L & =t^{2}\left(\partial^{2}+\left(p_{1} \hat{w}+p_{0}\right) \partial+q_{2} \hat{w}^{2}+q_{1} \hat{w}+q_{0}\right) \\
& =t^{2}\left(\partial-\alpha_{1}(\hat{w}+1)-\alpha_{0}\right) \cdot\left(\partial-\beta_{1} \hat{w}-\beta_{0}\right)
\end{aligned}
$$


After equating the coefficients we have: $\alpha_{1}=-p_{1}-\beta_{1}, \alpha_{0}=-p_{0}+p_{1}+\beta_{1}-\beta_{0}$, and the single Riccati equation for $\widehat{\beta}=\beta_{0}+\beta_{1} \hat{w}$ splits into the three equations

$$
\begin{aligned}
0 & =\beta_{1}^{2}+p_{1} \beta_{1}+q_{2} \\
-\beta_{1}^{\prime} & =2 \beta_{0} \beta_{1}+p_{0} \beta_{1}+p_{1} \beta_{0}+q_{1} \\
-\beta_{0}^{\prime} & =\beta_{0}^{2}+p_{0} \beta_{0}+q_{0}
\end{aligned}
$$

So we have an overdetermined system in $\beta_{0}$ and $\beta_{1}$, and there should be some compatibility condition of vanishing of the obstruction to factorization.

\section{Generalized Sturm-Liouville operator}

4.1. The Khudaverdian-Voronov canonical operator pencil. Return temporarily to arbitrary dimension. We shall use one important feature of $\mathfrak{D e n s}(M)$, which is the invariant scalar product. This gives the possibility of considering (formal) adjoint and self-adjoint operators. First of all,

$$
\hat{w}^{*}=1-\hat{w}, \quad\left(\partial_{a}\right)^{*}=-\partial_{a}, \quad t^{*}=t, \quad(f(x))^{*}=f(x),
$$

(where $\left.\partial_{a}=\frac{\partial}{\partial x^{a}}\right)$.

In the sequel, we write all the formulas for ordinary manifolds and even operators on them, for simplicity. (Though we still use $D x$ as the notation for the coordinate volume element. It is possible to write the formulas for the super case too, see [16].)

For a first order operator of weight $\mu$ given by (9), we have

$$
L^{*}=t^{\mu}\left(-A^{a}(x) \partial_{a}-B(x) \hat{w}-\partial_{a} A^{a}(x)+(1-\mu) B(x)+C(x)\right) .
$$

Hence it cannot be self-adjoint unless $A^{a} \equiv 0$ and $B \equiv 0$ (when it is effectively a zero-order operator). It is anti-self-adjoint if $-C=C-\partial_{a} A^{a}-(1-\mu) B$. Note that $C=L(1)$. If there are no a priori conditions for $C$, then this simply defines $C$ in terms of $A^{a}$ and $B$, which are not subject to any constraints,

$$
C=\frac{1}{2}\left(\partial_{a} A^{a}-(1-\mu) B\right) .
$$

If however we consider the particular case of operators with $C \equiv 0$, i.e., derivations

$$
L=t^{\mu}\left(A^{a}(x) \partial_{a}+B(x) \hat{w}\right)
$$

then $L^{*}=-L$ if and only if

$$
\partial_{a} A^{a}=(1-\mu) B
$$

which has different meanings for $\mu=1$ and for general $\mu \neq 1$. In the first case, we have the invariant "divergence-free" condition $\partial_{a} A^{a}=0$ for the vector density $A=|D x| A^{a} \partial_{a}$ on $M$ (of weight 1) and no restrictions for $B$. In the second case, $B$ is defined by $A^{a}$, and we have operators of the form

$$
L=t^{\mu}\left(A^{a}(x) \partial_{a}+\frac{\partial_{a} A^{a}}{1-\mu} \hat{w}\right)
$$

which are interpreted as "generalized Lie derivatives". (This is part of classification of the derivations of the algebra $\mathfrak{D} \mathfrak{e n s}(M)$ obtained in [16, 18].)

For second-order self-adjoint operators on $\mathfrak{D e n s}(M)$, the analysis was done in [16] (see also [18]). 
Theorem 1 ([16]). A self-adjoint second-order operator $L$ on the algebra of densities $\mathfrak{D e n s}(M)$ satisfying the condition $L(1)=0$ (annihilating constants) is completely defined by the following data:

(1) a tensor density $S=|D x|^{\mu} S^{a b} \partial_{a} \otimes \partial_{b}$ (the principal symbol of the restriction of $L$ on functions);

(2) an object $\gamma^{a}$ interpreted as "upper connection" coefficients, associated with $S$;

(3) an object $\theta$ called "Brans-Dicke field" (a terminology from physics), associated with $S$ and $\gamma^{a}$.

The operator defined by such data is given by

$$
L=t^{\mu}\left(S^{a b} \partial_{a} \partial_{b}+\left(2 \gamma^{a} \hat{w}+\partial_{a} S^{a b}+(\mu-1) \gamma^{b}\right) \partial_{b}+\theta \hat{w}^{2}+\left(\partial_{a} \gamma^{a}+(\mu-1) \theta\right) \hat{w}\right) .
$$

The symmetric matrix

$$
\hat{S}^{\hat{a} \hat{b}}=t^{\mu}\left(\begin{array}{cc}
S^{a b} & \gamma^{a} \\
\gamma^{b} & \theta
\end{array}\right)
$$

gives the principal symbol of $L$ (as a quadratic form) on the extended manifold $\hat{M}$.

(Grouping $S^{a b}, \gamma^{a}, \theta$ into the above matrix is the explanation of why the operator $L$ is parameterized this way. The theorem says, in brief: a second-order self-adjoint operator on the algebra $\mathfrak{D e n s}(M)$ annihilating constants is completely defined by its principal symbol.)

The coefficients $S^{a b}, \gamma^{a}, \theta$ as geometric objects on $M$ are characterized by their transformation laws under a change of coordinates: in "new" coordinates indicated by dash,

$$
\begin{aligned}
S^{a^{\prime} b^{\prime}} & =S^{a b} \frac{\partial x^{a^{\prime}}}{\partial x^{a}} \frac{\partial x^{b^{\prime}}}{\partial x^{b}}|J|^{\mu} \\
\gamma^{a^{\prime}} & =\left(\gamma^{a}-S^{a b} \partial_{b} \ln |J|\right) \frac{\partial x^{a^{\prime}}}{\partial x^{a}}|J|^{\mu} \\
\theta^{\prime} & =\left(\theta-2 \gamma^{a} \partial_{a} \ln |J|+S^{a b} \partial_{a} \ln |J| \cdot \partial_{b} \ln |J|\right)|J|^{\mu} .
\end{aligned}
$$

Here $J=\operatorname{Ber} \frac{\partial x}{\partial x^{\prime}}$.

Example 3. Objects of type $\gamma^{a}$ and $\theta$ can be constructed from a connection in the bundle of volume forms on $M$, as follows:

$$
\begin{array}{r}
\gamma^{a}:=S^{a b} \gamma_{a} \\
\theta:=S^{a b} \gamma_{a} \gamma_{b} .
\end{array}
$$

(Here $\gamma_{a}$ are the connection coefficients.) If $S^{a b}$ is invertible, then conversely, a connection $\gamma_{a}$ can be obtained from $\gamma^{a}$. But there will still be a freedom in defining $\theta$. (For a full analysis of the degrees of freedom there, see [16, 17] and [15].)

A self-adjoint operator $L$ on the algebra $\mathfrak{D e n s}(M)$ defined by (19) and specified by data $S^{a b}, \gamma^{a}, \theta$ is called canonical operator. (Its canonicity is in the fact that it requires less data than a general second-order operator and these data have a geometric meaning.) The corresponding quadratic operator pencil

$$
L_{w}=|D x|^{\mu}\left(S^{a b} \partial_{a} \partial_{b}+\left(2 \gamma^{a} w+\partial_{a} S^{a b}+(\mu-1) \gamma^{b}\right) \partial_{b}+\theta w^{2}+\left(\partial_{a} \gamma^{a}+(\mu-1) \theta\right) w\right)
$$

( $w$ is the parameter) is referred to as a canonical pencil. 
4.2. Generalized Sturm-Liouville operator. It is classically well-known (see [10]) that the familiar Sturm-Liouville operator $\partial^{2}+u(x)$ on the line can be uniquely described by the requirements that it keeps its form under a change of coordinate and is self-adjoint. (In particular, keeping the form without the first derivative implies that it cannot be regarded as acting on functions.) Together these conditions force it to be of the following form:

$$
L_{-\frac{1}{2}}=|d x|^{2}\left(\partial^{2}+u(x)\right): \quad \mathfrak{D e n s}_{-\frac{1}{2}} \rightarrow \mathfrak{D e n s}_{\frac{3}{2}} .
$$

(We use $d x$ instead of $D x$ for the case of the line.)

Comparison with the above indicates that this classical operator should be seen as the restriction of a canonical pencil on the line. This was first observed in [16, §4.1]. We can elaborate that as follows.

Proposition 1. The form of a second order operator $L$ of weight 2 on the algebra $\mathfrak{D e n s}(\mathbb{R})$,

$$
L=|d x|^{2}\left(\partial^{2}+\gamma(2 \hat{w}+1) \partial+\left(\theta(\hat{w}+1)+\gamma_{x}\right) \hat{w}\right),
$$

is invariant under changes of the independent variable $x$. It is uniquely defined by the conditions for $L$ being monic (i.e., the coefficient 1 at $\partial^{2}$ ), self-adjoint and annihilating constants. The operator $L$ is specified by geometric quantities $\gamma$ and $\theta$ and specializes to the classical Sturm-Liouville operator at $w=-1 / 2$.

Proof. Indeed, if we specialize formulas (19) and (24) for 1D, we shall obtain

$$
L=t^{\mu}\left(S \partial^{2}+(2 \gamma \hat{w}+\partial S+(\mu-1) \gamma) \partial+\theta \hat{w}^{2}+(\partial \gamma+(\mu-1) \theta) \hat{w}\right),
$$

and

$$
L_{w}=|d x|^{\mu}\left(S \partial^{2}+(2 \gamma w+\partial S+(\mu-1) \gamma) \partial+\theta w^{2}+(\partial \gamma+(\mu-1) \theta) w\right)
$$

for the corresponding operator pencil. Here $\partial=\partial_{x}$ (differentiation in the single variable), $\partial S=\partial(S)$, and $\partial \gamma=\partial(\gamma))$. The transformation of the coefficients $S, \gamma, \theta$ simplifies and takes the form:

$$
\begin{aligned}
& S^{\prime}=S J^{\mu-2} \\
& \gamma^{\prime}=(\gamma-S \partial \ln J) J^{\mu-1} \\
& \theta^{\prime}=\left(\theta-2 \gamma \partial \ln J+S(\partial \ln J)^{2}\right) J^{\mu} .
\end{aligned}
$$

We have assumed that $J>0$ and have therefore replaced $|J|$ by $J$, which in this $1 \mathrm{D}$ case coincides with the derivative $\frac{d x}{d x^{\prime}}$. It will be clarifying to write the derivatives such as $\partial f$ as $f_{x}$ indicating the variable explicitly and to elaborate further $\partial \ln J$. Since $J=\frac{d x}{d x^{\prime}}$, we rewrite it via $J^{-1}=\frac{d x^{\prime}}{d x}=x_{x}^{\prime}$. Finally, we obtain:

$$
\begin{aligned}
& S^{\prime}=S\left(x_{x}^{\prime}\right)^{2-\mu} \\
& \gamma^{\prime}=\left(\gamma x_{x}^{\prime}+S x_{x x}^{\prime}\right)\left(x_{x}^{\prime}\right)^{-\mu} \\
& \theta^{\prime}=\left(\theta\left(x_{x}^{\prime}\right)^{2}+2 \gamma x_{x}^{\prime} x_{x x}^{\prime}+S\left(x_{x x}^{\prime}\right)^{2}\right)\left(x_{x}^{\prime}\right)^{-\mu-2} .
\end{aligned}
$$

In particular, if the operator $L$ has weight $\mu=2$, the top coefficient $S$ becomes invariant. (A peculiarity of 1D.) We may choose $S$ to be 1 and hence arrive at (26). Clearly, at 
$w=-1 / 2$ the term with the first derivative vanishes and we obtain an operator of the classical Sturm-Liouville form.

We shall call such an operator $L$ on $\mathfrak{D e n s}(\mathbb{R})$ given by (26), the generalized SturmLiouville operator. The coefficients $\gamma$ and $\theta$ of the generalized Sturm-Liouville operator transform by

$$
\begin{aligned}
\gamma^{\prime} & =\left(\gamma x_{x}^{\prime}+x_{x x}^{\prime}\right)\left(x_{x}^{\prime}\right)^{-2} \\
\theta^{\prime} & =\left(\theta\left(x_{x}^{\prime}\right)^{2}+2 \gamma x_{x}^{\prime} x_{x x}^{\prime}+\left(x_{x x}^{\prime}\right)^{2}\right)\left(x_{x}^{\prime}\right)^{-4}
\end{aligned}
$$

The restriction of $L$ on $\mathfrak{D e n s}_{-\frac{1}{2}}$ gives the classical Sturm-Liouville operator (25) mapping $\mathfrak{D e n s}_{-\frac{1}{2}}$ to $\mathfrak{D e n s}_{\frac{3}{2}}$, with the potential $u=u(x)$ expressed via $\gamma, \theta$ by

$$
u=-\frac{1}{2}\left(\gamma_{x}+\frac{1}{2} \theta\right) \text {. }
$$

Proposition 2. The transformation laws for $\gamma$ and $\theta$ induce the well-known transformation law for the potential $u$,

$$
u^{\prime}=\left(u-\frac{1}{2} \mathfrak{S}_{x} x^{\prime}\right)\left(x_{x}^{\prime}\right)^{-2},
$$

where $\mathfrak{S}_{x} f$ denotes the Schwarzian derivative of a function $f$ with respect to a variable $x$.

Proof. Consider the transformation laws for $\gamma$ and $\theta$ given by (35) and (36). By differentiating both sides of (35) with respect to $x^{\prime}$, we obtain

$$
\gamma_{x^{\prime}}^{\prime}=\left(\gamma_{x}\left(x_{x}^{\prime}\right)^{2}-\gamma x_{x}^{\prime} x_{x x}^{\prime}+x_{x x x}^{\prime} x_{x}^{\prime}-2\left(x_{x x}^{\prime}\right)^{2}\right)\left(x_{x}^{\prime}\right)^{-4}
$$

Combining this with (36), we obtain directly

$$
\begin{aligned}
& \gamma_{x^{\prime}}^{\prime}+\frac{1}{2} \theta^{\prime}=\left(\gamma_{x}\left(x_{x}^{\prime}\right)^{2}-\gamma x_{x}^{\prime} x_{x x}^{\prime}+x_{x x x}^{\prime} x_{x}^{\prime}-2\left(x_{x x}^{\prime}\right)^{2}+\frac{1}{2} \theta\left(x_{x}^{\prime}\right)^{2}+\gamma x_{x}^{\prime} x_{x x}^{\prime}+\frac{1}{2}\left(x_{x x}^{\prime}\right)^{2}\right)\left(x_{x}^{\prime}\right)^{-4}= \\
& \left(\left(\gamma_{x}+\frac{1}{2} \theta\right)\left(x_{x}^{\prime}\right)^{2}+x_{x x x}^{\prime} x_{x}^{\prime}-\frac{3}{2}\left(x_{x x}^{\prime}\right)^{2}\right)\left(x_{x}^{\prime}\right)^{-4}=\left(\gamma_{x}+\frac{1}{2} \theta+\frac{x_{x x x}^{\prime}}{x_{x}^{\prime}}-\frac{3}{2}\left(\frac{x_{x x}^{\prime}}{x_{x}^{\prime}}\right)^{2}\right)\left(x_{x}^{\prime}\right)^{-2},
\end{aligned}
$$

which is exactly

$$
\gamma_{x^{\prime}}^{\prime}+\frac{1}{2} \theta^{\prime}=\left(\gamma_{x}+\frac{1}{2} \theta+\mathfrak{S}_{x} x^{\prime}\right)\left(x_{x}^{\prime}\right)^{-2},
$$

by the definition of the Schwarzian derivative:

$$
\mathfrak{S}_{x} f=\frac{f_{x x x}}{f_{x}}-\frac{3}{2}\left(\frac{f_{x x}}{f_{x}}\right)^{2}
$$

for a function $f=f(x)$ (see, e.g. [20]). This gives (38) .

(The appearance of the Schwarzian derivative in the transformation of the potential for the Sturm-Liouville equation is classical, see e.g. [10, §3.8]. Its geometric interpretation can be found in [20]. The fact that this transformation law is a corollary for the $1 \mathrm{D}$ case of the transformation laws of an "upper connection" on volume forms $\gamma^{a}$ and "Brans-Dicke field" $\theta$ was discovered in [16].) 
4.3. Factorization of the generalized Sturm-Liouville operator. In Sec. 2.4 we observed that when modules of differential operators acting on densities of non-singular weights are studied, the families of operators that appear correspond to self-adjoint operators on the algebra of densities. There is one per family self-adjoint operator acting between densities of a particular combination of weights [16]. It corresponds to a singular value of the parameter. Now, of course, operators on the algebra of densities can be specialized to operators acting on densities of singular weights, just there will be no one-to-one correspondence.

Consider (26) , the generalized Sturm-Liouville operator. When specialized on $\mathfrak{D e n s}-1 / 2$, it becomes the classical Sturm-Liouville operator $t^{2}\left(\partial^{2}+u\right)$ with the potential

$$
u=-\frac{1}{2}\left(\gamma_{x}+\frac{\theta}{2}\right)
$$

as we have just discussed. Rewriting everything in terms of $u$ instead of $\theta$ and an operator $\hat{\lambda}$ such that $\hat{w}=-\frac{1}{2}+\hat{\lambda}$, we come to

$$
L=t^{2}\left(\partial^{2}+2 \gamma \hat{\lambda} \partial-2\left(\gamma_{x}+2 u\right) \hat{\lambda}^{2}+\gamma_{x} \hat{\lambda}+u\right)
$$

where the classical Sturm-Liouville now corresponds to $\lambda=0$.

Theorem 2. The necessary and sufficient condition for the generalized Sturm-Liouville operator $L$ given by (26) or (39) to be factorizable is that the quantity $\psi$ defined by

$$
\psi:=\left(\gamma^{2}+2\left(\gamma^{\prime}+2 u\right)\right)^{-1 / 4}
$$

satisfies the classical Sturm-Liouville equation

$$
\left(\partial^{2}+u\right) \psi=0 \text {. }
$$

Proof. Consider the factorization problem $L=t^{2}(\partial-\widehat{\alpha})(\partial-\widehat{\beta})$, which, according to the general analysis in the previous section, is parameterized by $\widehat{\beta}=b_{0}+b_{1} \hat{\lambda}$ and the remaining conditions are

$$
\begin{aligned}
-b_{0}^{\prime} & =b_{0}^{2}+u \\
-b_{1}^{\prime} & =2 b_{0} b_{1}+2 \gamma b_{0}+\gamma^{\prime} \\
0 & =b_{1}^{2}+2 \gamma b_{1}-2\left(\gamma^{\prime}+2 u\right)
\end{aligned}
$$

(here prime is used for the derivative). From the third equation we get:

$$
b_{1}+\gamma= \pm \sqrt{\gamma^{2}+2\left(\gamma^{\prime}+2 u\right)} \text {. }
$$

$b_{1}+\gamma$ is exactly what we need for the second equation, which has the form

$$
-\left(b_{1}+\gamma\right)^{\prime}=2 b_{0}\left(b_{1}+\gamma\right) \text {. }
$$

But first let us solve (43) for $b_{0}$ (and then substitute the expression from (42)):

$$
\begin{aligned}
b_{0}=-\frac{1}{2} \partial \ln \left(b_{1}+\gamma\right)=-\frac{1}{2} \frac{\left( \pm \sqrt{\gamma^{2}+2\left(\gamma^{\prime}+2 u\right)}\right)^{\prime}}{ \pm \sqrt{\gamma^{2}+2\left(\gamma^{\prime}+2 u\right)}}= \\
\quad-\frac{1}{2} \partial \ln \sqrt{\gamma^{2}+2\left(\gamma^{\prime}+2 u\right)}=\partial \ln \left(\gamma^{2}+2\left(\gamma^{\prime}+2 u\right)\right)^{-1 / 4} .
\end{aligned}
$$


Denoting

$$
\psi:=\left(\gamma^{2}+2\left(\gamma^{\prime}+2 u\right)\right)^{-1 / 4}
$$

the previous expression for $b_{0}$ becomes $b_{0}=\partial \ln \psi$. What is left, is the first equation from the original system, which is the "classical" Riccati equation:

$$
-b_{0}^{\prime}=b_{0}^{2}+u,
$$

equivalent to $\psi$ satisfying the Sturm-Liouville equation with the potential $u$.

One immediate question concerns invariance of the obtained condition under a change of coordinates. For that we need to consider the transformation law for the object $\psi$. Note that if we express back $u$ via $\gamma, \theta$, then the expression for $\psi$ takes the form

$$
\psi=\left(\gamma^{2}-\theta\right)^{-1 / 4} \text {. }
$$

Theorem 3. The $-\frac{1}{2}$-density

$$
\boldsymbol{\psi}:=\left(\gamma^{2}-\theta\right)^{-1 / 4}|d x|^{-1 / 2}
$$

does not depend on a change of coordinate and hence is an invariant of the generalized Sturm-Liouville operator L.

Proof. We need to recall the transformation laws (35) and (36). We return to the notation where prime is used for "new" variables and quantities in new variables. We shall again use subscripts for derivatives. From (35) and (36), we have

$$
\begin{aligned}
\left(\gamma^{\prime}\right)^{2} & =\left(\gamma^{2}\left(x_{x}^{\prime}\right)^{2}+2 \gamma x_{x}^{\prime} x_{x x}^{\prime}+\left(x_{x x}^{\prime}\right)^{2}\right)\left(x_{x}^{\prime}\right)^{-4}, \\
\theta^{\prime} & =\left(\theta\left(x_{x}^{\prime}\right)^{2}+2 \gamma x_{x}^{\prime} x_{x x}^{\prime}+\left(x_{x x}^{\prime}\right)^{2}\right)\left(x_{x}^{\prime}\right)^{-4},
\end{aligned}
$$

and by subtracting we obtain after simplification

$$
\gamma^{\prime 2}-\theta^{\prime}=\left(\gamma^{2}-\theta\right)\left(x_{x}^{\prime}\right)^{-2} .
$$

Hence the quantity $\boldsymbol{\omega}=\left(\gamma^{2}-\theta\right)|d x|^{2}$ is invariant. It is a well-defined density of weight 2 . Therefore the quantity

$$
\boldsymbol{\psi}=\psi|d x|^{-1 / 2}=\boldsymbol{\omega}^{-1 / 4}
$$

is a well-defined density of weight $-1 / 2$.

By the properties of the classical Sturm-Liouville operator, the condition that $\boldsymbol{\psi}$ is a solution does not depend on a choice of coordinate. This establishes the invariance of the factorization criterion.

For the same operator $L$ on the algebra $\mathfrak{D} \mathfrak{e n s}(\mathbb{R})$, we can pose the problem of an "incomplete factorization":

$$
L=t^{2}((\partial-\widehat{\alpha})(\partial-\widehat{\beta})+f)
$$

where $f=f(x)$ does not contain $\hat{w}$. This gives the system

$$
\begin{aligned}
-b_{0}^{\prime} & =b_{0}^{2}+u-f \\
-b_{1}^{\prime} & =2 b_{0} b_{1}+2 \gamma b_{0}+\gamma^{\prime} \\
0 & =b_{1}^{2}+2 \gamma b_{1}-2\left(\gamma^{\prime}+2 u\right)
\end{aligned}
$$


for the unknowns $b_{0}, b_{1}$ and $f$. A slight modification of the argument above leads us to the following theorem.

Theorem 4. An incomplete factorization of the generalized Sturm-Liouville operator is always possible and is unique. It is given by the formulas

$$
\begin{aligned}
b_{0} & =\partial \ln \psi, \\
b_{1} & =-\gamma \pm \frac{1}{\psi^{2}}, \\
f & =\frac{1}{\psi}\left(\partial^{2}+u\right) \psi .
\end{aligned}
$$

Here $\psi$ is as above. (We assume that $\psi$ is not identically zero.)

One can draw some indirect analogy for these results with the Laplace (differential) gauge invariants $h$ and $k$, which appear in the study of operators of the form $\partial_{x} \partial_{y}+a \partial_{x}+b \partial_{y}+c$ in the classical setting, the direction originally started in Darboux's famous book [4]. The $h$ and $k$ are invariants relative to gauge transformations of the operator and are a complete system of invariants. They were generalized for dimension three in the recent work [1]. It would also be interesting to analyze in a similar way the case of differential operators on the algebra $\mathfrak{D e n s}\left(\mathbb{R}^{1 \mid 1}\right)$ for the superline. Compare [19].

\section{ACKNOWLEDGEMENT}

This material is based upon work supported by the National Science Foundation under Grant No.1708033. We are very thankful to Yuri Berest for the stimulating discussion and providing a reference to the original publication containing the Frobenius theorem on factorization and the anonymous referee for remarks that helped to improve the exposition.

\section{REFERENCES}

[1] Ch. Athorne. Laplace maps and constraints for a class of third-order partial differential operators. Journal of Physics A: Mathematical and Theoretical, 51(8), 2018.

[2] A. Biggs. The existence of a canonical lifting of even Poisson structures to the algebra of densities. Lett. Math. Phys., 104(12):1523-1533, 2014.

[3] A. Biggs and H. Khudaverdyan. Operator pencil passing through a given operator. Journal of Mathematical Physics, 54(12), 2013.

[4] G. Darboux. Leçons sur la théorie générale des surfaces et les applications géométriques du calcul infinitésimal, volume 2. Gauthier-Villars, 1889.

[5] C. Duval and V. Yu. Ovsienko. Space of second-order linear differential operators as a module over the Lie algebra of vector fields. Adv. Math., 132(2):316-333, 1997.

[6] G. Frobenius. Ueber die Determinante mehrerer Functionen einer Variabeln. J. Reine Angew. Math., $77: 245-257,1874$.

[7] H. Gargoubi, N. Mellouli, and V. Ovsienko. Differential operators on supercircle: conformally equivariant quantization and symbol calculus. Lett. Math. Phys., 79(1):51-65, 2007.

[8] J. George. Projective connections and the algebra of densities. In Geometric methods in physics, volume 1079 of AIP Conf. Proc., pages 142-148. Amer. Inst. Phys., Melville, NY, 2008.

[9] S. Hill, E. Shemyakova, and Th. Voronov. Darboux transformations for differential operators on the superline. Russian Math. Surveys, 70(6):1173, 2015. arXiv:1505.05194 [math.MP].

[10] N. J. Hitchin, G. B. Segal, and R. S. Ward. Integrable systems, volume 4 of Oxford Graduate Texts in Mathematics. The Clarendon Press, Oxford University Press, New York, 2013. Twistors, loop 
groups, and Riemann surfaces, Lectures from the Instructional Conference held at the University of Oxford, Oxford, September 1997, Paperback reprint [of MR1723384].

[11] D. Hobby and E. Shemyakova. Classification of multidimensional Darboux transformations: first order and continued type. SIGMA (Symmetry, Integrability and Geometry: Methods and Applications), 13(10):20 pages, 2017. arXiv:1605.04362 [math.DG].

[12] O. Khudaverdian. Geometry of superspace with even and odd brackets. J. Math. Phys., 32(7):19341937, 1991. Preprint of the Geneva University, UGVA-DPT 1989/05-613.

[13] H. Khudaverdian. Delta-Operator on Semidensities and Integral Invariants in the Batalin-Vilkovisky Geometry. Preprint 1999/135, Max-Planck-Institut fuer Mathematik Bonn, 1999.

[14] H. M. Khudaverdian. Semidensities on odd symplectic supermanifolds. Comm. Math. Phys., $247(2): 353-390,2004$.

[15] H. Khudaverdian and M. Peddie. Odd Laplacians: geometrical meaning of potential and modular class. Lett. Math. Phys., 107(7):1195-1214, 2017.

[16] H. Khudaverdian and Th. Voronov. On odd Laplace operators. II. In Geometry, topology, and mathematical physics, volume 212 of Amer. Math. Soc. Transl. Ser. 2, pages 179-205. Amer. Math. Soc., Providence, RI, 2004.

[17] H. Khudaverdian and Th. Voronov. Geometry of differential operators of second order, the algebra of densities, and groupoids. J. Geom. Phys., 64:31-53, 2013.

[18] H. Khudaverdian and Th. Voronov. Geometric constructions on the algebra of densities. In Topology, geometry, integrable systems, and mathematical physics, volume 234 of Amer. Math. Soc. Transl. Ser. 2, pages 241-263. Amer. Math. Soc., Providence, RI, 2014.

[19] S. Li, E. Shemyakova, and Th. Voronov. Differential operators on the superline, Berezinians, and Darboux transformations. Lett. Math. Phys., 107(9):1689-1714, 2017.

[20] V. Ovsienko and S. Tabachnikov. Projective differential geometry old and new. From the Schwarzian derivative to the cohomology of diffeomorphism groups, volume 165 of Cambridge Tracts in Mathematics. Cambridge University Press, Cambridge, 2005.

[21] A. Schwarz. Geometry of Batalin-Vilkovisky quantization. Comm. Math. Phys., 155(2):249-260, 1993.

[22] E. Shemyakova. Invertible Darboux transformations. SIGMA (Symmetry, Integrability and Geometry: Methods and Applications), 9:Paper 002, 10, 2013.

[23] E. Shemyakova. Proof of the completeness of Darboux Wronskian formulae for order two. Canad. J. Math., 65(3):655-674, 2013.

[24] E. Shemyakova. Factorization of Darboux transformations of arbitrary order for 2D Schrödinger operator. submitted, 2017. arXiv:1304.7063 [math.MP].

[25] T. Thomas. A projective theory of affinely connected manifolds. Math. Z., 25(1):723-733, 1926.

Department of Mathematics, University of Toledo, Toledo, Ohio, USA

E-mail address: ekaterina.shemyakova@utoledo.edu

School of Mathematics, University of Manchester, Manchester, UK

E-mail address: theodore.voronov@manchester.ac.uk

Faculty of Physics, Tomsk State University, Tomsk, 634050, Russia 